\title{
A New Approach in Coal Mine Exploration Using Cosmic Ray Muons
}

\author{
Reza DARIJANI ${ }^{1}$, Ali NEGARESTANI ${ }^{2}$, Mohammad Reza REZAIE ${ }^{2}$, \\ Syed Jalil FATEMI ${ }^{3}$, and Ahmad AKHOND ${ }^{1}$ \\ ${ }^{1}$ Department of Physics, Payame Noor University, Tehran, Iran \\ ${ }^{2}$ Department of Physics, Kerman Graduate University of Technology, Kerman, Iran \\ e-mail: mr.rezaie@kgut.ac.ir (corresponding author) \\ ${ }^{3}$ Department of Physics, Shahid Bahonar University, Kerman, Iran
}

\begin{abstract}
Muon radiography is a technique that uses cosmic ray muons to image the interior of large scale geological structures. The muon absorption in matter is the most important parameter in cosmic ray muon radiography. Cosmic ray muon radiography is similar to X-ray radiography. The main aim in this survey is the simulation of the muon radiography for exploration of mines. So, the production source, tracking, and detection of cosmic ray muons were simulated by MCNPX code. For this purpose, the input data of the source card in MCNPX code were extracted from the muon energy spectrum at sea level. In addition, the other input data such as average density and thickness of layers that were used in this code are the measured data from Pabdana (Kerman, Iran) coal mines. The average thickness and density of these layers in the coal mines are from 2 to $4 \mathrm{~m}$ and $1.3 \mathrm{gr} / \mathrm{cm}^{3}$, respectively. To increase the spatial resolution, a detector was placed inside the mountain. The results indicated that using this approach, the layers with minimum thickness about $2.5 \mathrm{~m}$ can be identified.
\end{abstract}

Key words: muon radiography, coal mines, MCNPX.

Ownership: Institute of Geophysics, Polish Academy of Sciences;

(C) 2016 Darijani et al. This is an open access article distributed under the Creative Commons Attribution-NonCommercial-NoDerivs license,

http://creativecommons.org/licenses/by-nc-nd/3.0/. 


\section{INTRODUCTION}

When the primary cosmic ray particles, protons and $\alpha$ particles, penetrate the atmosphere they collide with air nuclei and new particles, mostly pions and kaons are produced. These secondary particles are short lived, and a fraction of them decay into muons instead of interacting with nuclei deeper in the atmosphere. Because these cosmic ray muons weakly interact with air nuclei, and their mean lifespan is long (about $2.2 \times 10^{-6} \mathrm{~s}$ ), they are the dominant charged particles at the Earth s surface. They arrive at angles ranging from vertical to horizontal (Thompson and Whalley 1975), and their energy spectrum has been measured as a function of the zenith angle $\theta$ (Allkofer $e t$ al. 1981). It is also well known that muons arriving in the horizontal direction have a higher intensity at energies above $100 \mathrm{GeV}$. Because muons become highly penetrative as their energy becomes higher, these horizontal muons can be used for the purpose of probing the internal structure of gigantic objects. For example, typical horizontally arriving cosmic ray muons with energy of $1 \mathrm{TeV}$ penetrate $2.6 \mathrm{~km}$ of water (Tanaka et al. 2007). Since muons penetrate through matter their energy decreases. The energy loss depends on the kinetic energy of the muon and, at very high energies, also on the chemical composition of the matter (Malmqvist et al. 1979). Some of muons that do not have enough energy to escape from the matter of interest are absorbed in the matter, and as a result the muon flux is reduced. Absorption of muons in matter is independent of geophysical models and directly measures the density length. Geophysical models can be used to predict a host of geophysical measurements and are important ways of calibrating regions in the absence of direct measurements. Meanwhile, geophysical models explain geophysical observations and determine the subsurface Earth properties. By measuring cosmic ray muons intensity in different depths of matter, very useful information regarding the density of matter can be obtained. This information is used for many purposes such as exploration of mines, and prediction of volcanic eruptions and earthquakes. The aim of this research is to obtain a two-dimensional profile of the average density inside a mountain by simulation. To this end, production, tracking, and detection of muons were performed by the MCNPX code. The input data such as the average density and thickness of layers that have been used in MCNPX code are the measured data from Pabdana (Kerman, Iran) coal mines.

\section{THEORY}

The potential use of high-energy near-horizontal cosmic ray muons for exploring the internal structure of large scale objects such as Egyptian pyramids has been recognized (Alvarez et al. 1970). The effective use of such near-horizontal cosmic ray muons for muon radiography can be explained through the following steps. 


\subsection{The cosmic-ray muon energy spectrum and its dependence on zenith angle}

Pions and kaons are produced through nuclear interactions between primary cosmic ray protons and the atmospheric air, some of which decay and produce cosmic ray muons. The energy spectrum of these cosmic ray muons has been measured at different polar angles (Thompson and Whalley 1975). Furthermore, the flux of incident cosmic muons is of critical importance for geophysical imaging since it is used to determine the attenuation produced by the geological target (Lesparre et al. 2010). There are two ways to derive the differential flux of muons. The first approach is the use of a full simulation. These Monte Carlo computations can be accomplished by simulation codes like CORSIKA which allows to take into account the geomagnetic and altitude dependence. The second approach to obtain flux models is the use of fitting. The choice of a particular parameterization of the fitting curves may either be inspired by the physics involved in the production of muons from their parents or be guessed to provide a better fit regardless of the physical meaning of the parameters (Gaisser 1990). Some of fitting models only consider the production of muons from pions and kaons decay and assume a primary proton flux of the form: $P_{0} e^{-\gamma}$ with $P_{0}=1.8\left(\mathrm{~cm}^{-2} \mathrm{sr}^{-1} \mathrm{GeV}^{\gamma-1}\right)$, and $\gamma=2.7$. This approach yields the analytical form of the muon spectrum initially proposed by Bugaev et al. (1970) and popularized by Gaisser in the form of Eq. 1 (Lesparre et al. 2010).

$$
\varphi_{G}\left(\mathrm{E}_{0}, \theta\right)=A_{G} E_{0}^{-\gamma}\left(\frac{1}{1+\frac{E_{0} \cos \theta}{E_{0, \pi}^{\mathrm{cr}}}}+\frac{B_{G}}{1+\frac{E_{0} \cos \theta}{E_{0, \kappa}^{\mathrm{cr}}}}\right),
$$

where $A_{G}, \gamma$, and $B_{G}$ are the scale factor, the power index, and the balance factor, respectively. The balance factor depends on the ratio of muons produced by the kaons and pions. $E_{0, \pi}^{\mathrm{cr}}$ and $E_{0, \kappa}^{\mathrm{cr}}$ are critical energies of pions and kaons for the vertical incidence $(\theta=0)$.

\subsection{Range of cosmic ray muons through standard rock}

The energy loss of a charged particle with some energy $E(\mathrm{TeV})$ on passage though matter with a density length (density $\times$ path length) of $X$ hectogram per $\mathrm{cm}^{2}, X\left(\mathrm{hg} / \mathrm{cm}^{2}=100 \mathrm{gr} / \mathrm{cm}^{2}\right)$, can be written as (Tanaka et al. 2007, Nagamine et al. 1995). 


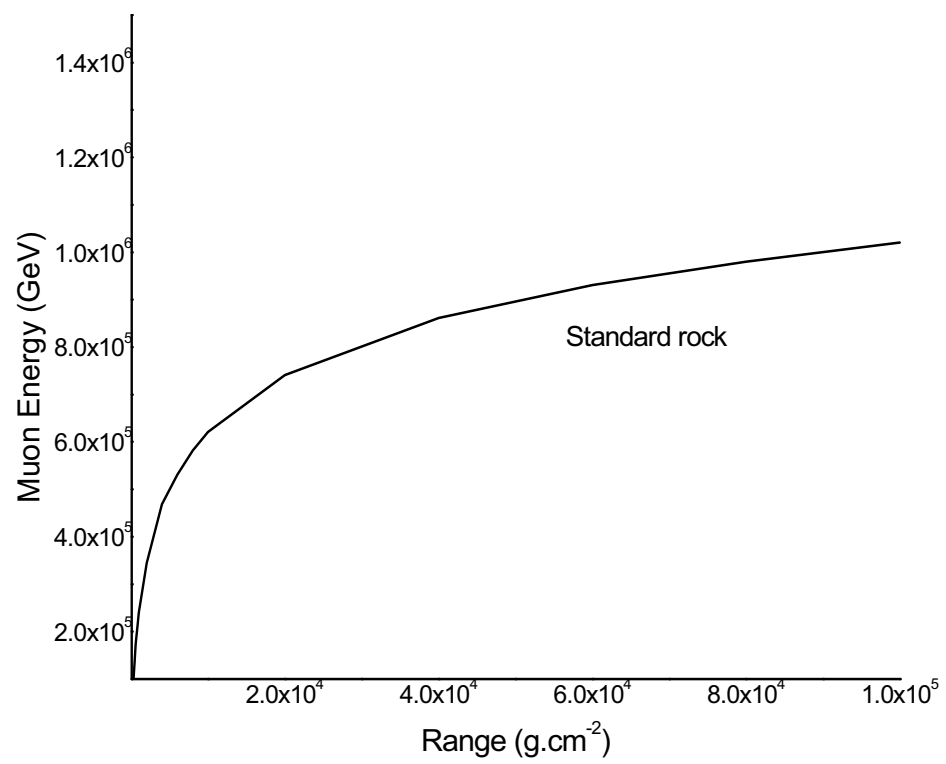

Fig. 1. The muon energy versus range in a standard rock.

$$
\frac{d E}{d X}=\left[1.88+0.077 \mathrm{Ln}\left(\frac{E}{m_{\mu}}\right)+3.9 E\right] \times 10^{-6}\left[\mathrm{TeVcm}^{2} / \mathrm{gr}\right],
$$

where the first two terms represent ionization loss and the third term represents stochastic processes mainly due to bremsstrahlung. Choosing $E=1 \mathrm{TeV}$ to yield a relatively small contribution of the logarithmic term, the mean rang (density length) $X$ can be obtained by integrating the energy loss formula as Eq. 3 (Nagamine et al. 1995, Nagamine 2003).

$$
X=2.5 \times 10^{3} \operatorname{Ln}(1.56 E+1)\left[\mathrm{hg} / \mathrm{cm}^{2}\right] .
$$

This equation gives the relationship between range and energy in a standard rock. Standard rock is defined by $z / A=0.5, z^{2} / A=5.5, Z=11$, and $\rho=2.56 \mathrm{gr} / \mathrm{cm}^{3}$, where $Z, A$, and $\rho$ are the mean atomic number, the mean mass number, and density of the rock, respectively. Figure 1 shows the muon energy versus range in a standard rock.

\subsection{Intensity of cosmic ray muons penetrating though rock with density length $X$}

Measurement of muons intensity at various depths is important from different aspects. For instance, it provides information on the electromagnetic processes that reduce the flux (Bektasoglu et al. 2012). The measured muons 
intensity at depth $x$ in the Earth is equal to the intensity of muons at sea level with an energy greater than $E_{c}(x)$; disregarding the small fraction of muons which decay in the passage from the ground to the depth $x, E_{c}(x)$ is the minimum energy necessary for a muon to penetrate to the depth $x$ (Malmqvist et al. 1979). Thus a unique relationship exists between density length $X$ $(X=\rho x)$ and the intensity of penetrating cosmic ray muons $N_{\mu}\left(E_{c}(x), \theta_{Z}\right)$. Once $X$ is given, the minimum energy $E_{c}(x)$ is determined through the $X-E$ relation (Eq. 3), and the integrated flux $N_{\mu}\left(E_{c}(x), \theta_{Z}\right)$ is given as Eq. 4.

$$
N_{\mu}\left(E_{c}, \theta=90-\theta_{z}\right)=\int_{E_{C}}^{\infty} \frac{d N}{d E} d E .
$$

Conversely, for a substance with an unknown density length $X$, the measurement of the muons flux, $N_{\mu}\left(\theta_{Z}\right)$, penetrating through the substance with a zenith angel, $\theta_{Z}$, uniquely determines its density length in $\mathrm{hg} / \mathrm{cm}^{2}$. Small changes in $X$ due to the existence of a region of lower or higher density inside the broadly uniform object lead to differences in $N_{\mu}\left(\theta_{Z}\right)$. The change in $N_{\mu}\left(\theta_{Z}\right)$ informs us of change in $X$ (Nagamine 2003). Figure 2 shows the penetrating muons flux as a function of rock thickness at different zenith angles.

Using Fig. 2, the thickness of rock (in $\mathrm{km}$ ) is calculated by dividing rock thickness (in $\mathrm{km}$ ) water equivalent by rock density.

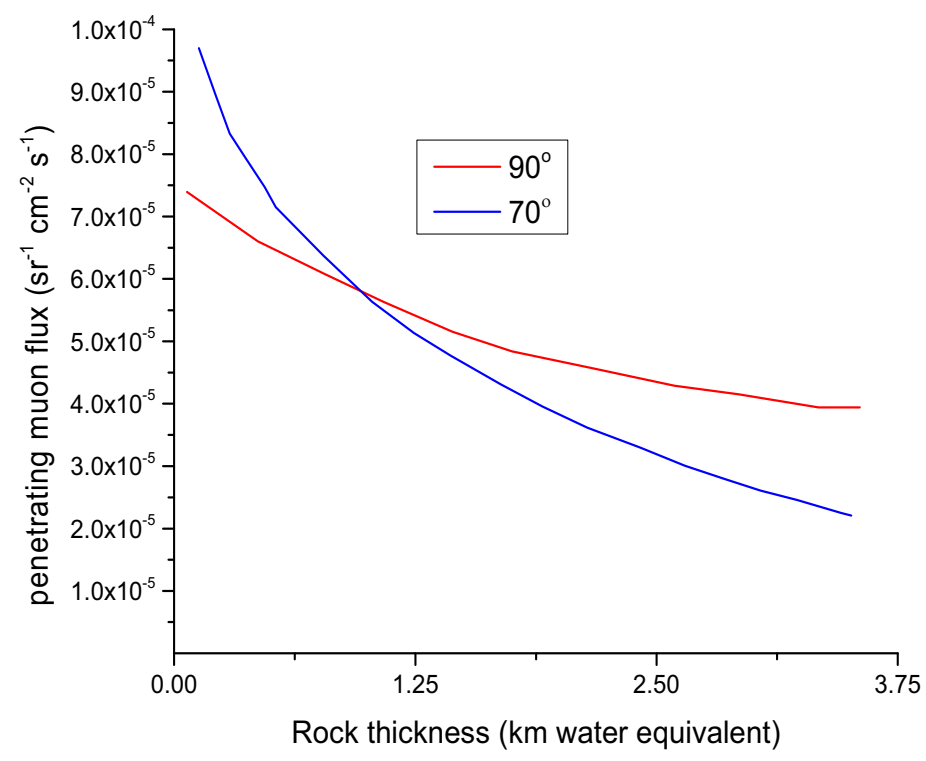

Fig. 2. Penetrating muon flux versus rock thickness (Nagamine 2003). 


\section{DETERMINATION COSMIC RAY MUON PATH THROUGH THE MATTER}

For determination of the path of the muon penetrating through gigantic objects, there are two types of practical detection systems that are explained in the following.

\subsection{Telescope of position sensitive detector}

In this case, the straight line connecting the points where a cosmic ray muon passes through two (or three) counters determines the particle path (Fig. 3).

There are two representative examples of position determination that are explained in the following:

- The passing points can be determined by the difference in the arrival time of scintillation light from a set of at least three photomultipliers attached to the edges of each scintillator.

- The passing points can be determined more straightly by using segmented counter arrays in both horizontal and vertical directions (Fig. 3b).

In the case of counter telescopes of the position sensitive detectors, the spatial resolution of the detection system at the mountain $(\Delta X, \Delta Y)$ can be determined from the resolution of the intersection points with each counter $(\Delta x$, $\Delta y)$, the distance between the counters comprising the telescope $(l)$, and the distance between the object (mountain) and the detector $(L)$. This spatial resolution is calculated by the following Eqs. 5 and 6 .
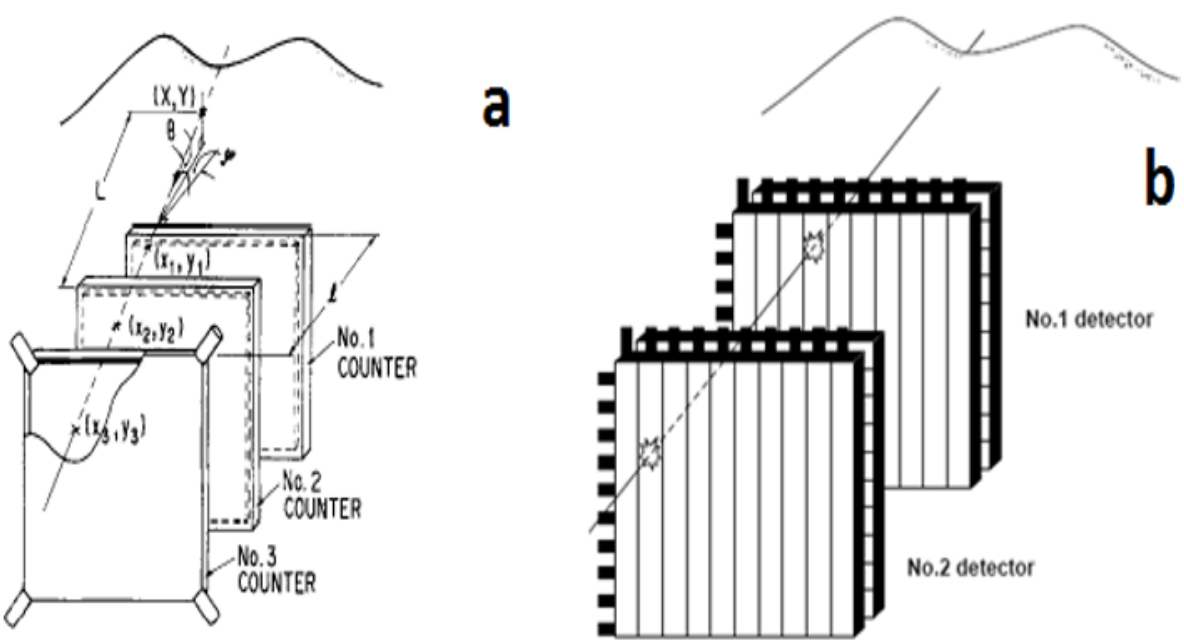

Fig. 3: (a) The counter telescope with three plastic scintillators, and (b) the detection system comprising two segmented plastic counters (Nagamine et al. 1995). 


$$
\begin{aligned}
& \Delta X=\frac{L}{l} \Delta x, \\
& \Delta Y=\frac{L}{l} \Delta y .
\end{aligned}
$$

\subsection{Cerenkov light detector}

In this case, the passage of the relativistic muon leaves a track of Cerenkov radiation in a selected gas or liquid which can be collected using a concave reflective mirror focused on an array of photomultipliers (Nagamine et al. 1995, Tanaka et al. 2003).

\section{KERMAN COAL MINES}

Kerman coal mines are situated in the east of central Iran at the distance between 50 and $220 \mathrm{~km}$ north west of Kerman. At these areas there are 9 coal ores that the Main Pabdana and the South Pabdana are active and important mines.

\subsection{Main Pabdana coal mine}

This coal mine is around $9 \mathrm{~km}$ long and $1.1 \mathrm{~km}$ wide, and its area is about $10 \mathrm{~km}^{2}$. It consists of 14 layers of coal which are located at different depths and have different thicknesses. Their maximum thickness is of about $4 \mathrm{~m}$, while for some others the thickness is about $2 \mathrm{~m}$. The average density of coal and that of rock in this mine are about 1.3 and $2.65 \mathrm{gr} / \mathrm{cm}^{3}$, respectively.

\subsection{The South Pabdana mine}

This mine like the Main Pabdana was explored from 1966 to 1977. Its area is about $2 \mathrm{~km}^{2}$. It consists of 13 layers, each one having an average thickness between 1 and $3.15 \mathrm{~m}$ and the average density of $1.3 \mathrm{gr} / \mathrm{cm}^{3}$.

\section{MCNPX CODE}

MCNPX is a general-purpose Fortran 90 Monte Carlo radiation transport code that transports more than 34 particle types including neutrons, protons, electrons, muons, etc. MCNPX is the product of computer codes that are used for modeling, the interaction of particle with matter, radiography, exploration of mine and oil, medical physics, and shielding. MCNPX has many capabilities that enhance physics, sources, tallies, and graphics (Pelowitz 2008) making the results of simulations more accurate. The simulation performed in the present study has many stages that are explained in the following.

\subsection{Geometry}

In this study geometry is defined as a cone that is $200 \mathrm{~m}$ high, parallel to the $z$-axis, and the opening angle of the cone is 45 degrees. This cone is inter- 


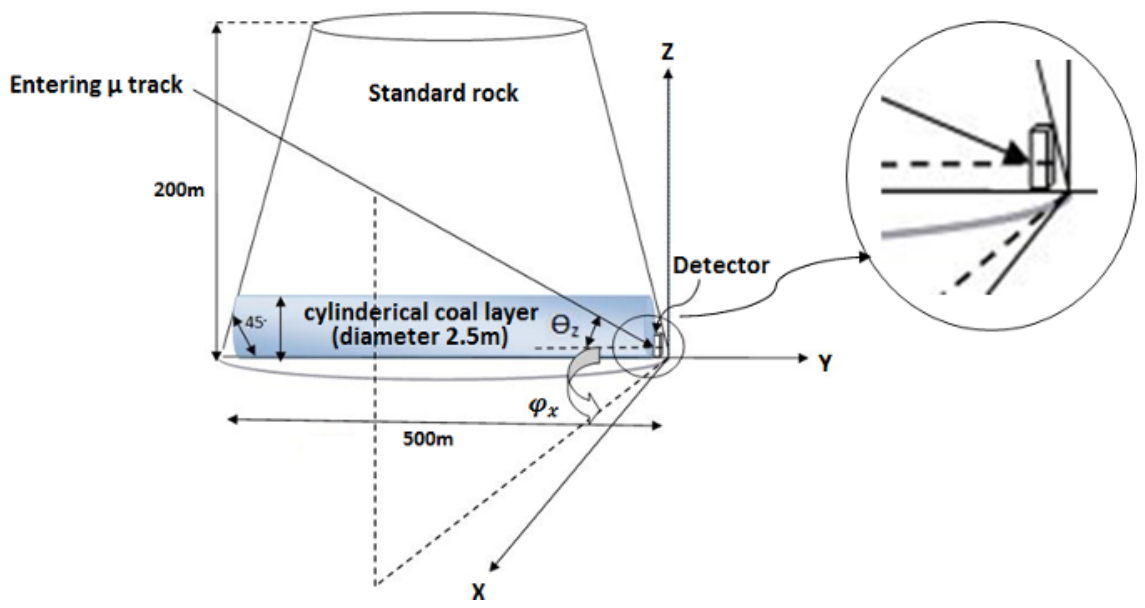

Fig. 4. Simplified model for mountain-detector and polar coordinate system.

sected by two planes parallel to $x y$ plane at $z=0$ and $z=200 \mathrm{~m}$, respectively. Inside this cone, there is a cylindrical layer of coal with diameter of $2.5 \mathrm{~m}$ which is located at a depth of $200 \mathrm{~m}$ from the mountain top (cone) with a width of $500 \mathrm{~m}$; the remaining volume of the cone is standard rock. The coal was replaced by carbon that is defined by $z / A=0.5, z^{2} / A=3$, $Z=6$, and $\rho=1.3 \mathrm{gr} / \mathrm{cm}^{3}$, where $Z, A$, and $\rho$ are the mean atomic number, the mean mass number, and density of the coal, respectively. A detection system with an area of about $1 \mathrm{~m}^{2}$, perpendicular to the $y$ axis, was located at the foot of cone. The geometry and detector that were used in MCNPX code have been shown in Fig. 4.

\subsection{Source card}

The source card of MCNPX code includes all necessary data for generation of particles (positive and negative muons) with energy spectrum at sea level. The energy distribution and life time of positive and negative muons are equal. But due to the fact that the primary cosmic rays, protons $(95 \%)$ and He $(5 \%)$, are essentially positively charged, the secondary component in the atmosphere has a positive charge excess (between 20 and 30\%), too. These data with energies ranging from 1 to $10^{3} \mathrm{GeV}$ were extracted using Eq. 1. In this equation, values of parameters $A_{G}, \gamma$, and $B_{G}$ are $0.14\left(\mathrm{~cm}^{-2} \mathrm{sr}^{-1} \mathrm{~s}^{-1} \mathrm{GeV}^{\gamma-1}\right)$, 2.7 , and 0.054 , respectively. In addition, the values of critical energies of pions and kaons, $E_{0, \pi}^{\mathrm{cr}}$ and $E_{0, \kappa}^{\mathrm{cr}}$, are $115 / 1.1$ and 850/1.1, respectively. Meanwhile, $E_{0}$ and $\theta$ are, respectively, the energy and zenith angle of incident cosmic ray muon. In this study, the source has been defined using general source and SDEF card for positive and negative muons separately. The main 
control keywords for the SDEF card are position, type, and energy of the particle.

\subsection{Tally (output)}

The appropriate MCNPX tally that is of event-by-event nature and fits the study requirement is the PTRAC card. The main control keywords for the PTRAC card are: (i) output file, (ii) event type filter, and (iii) history filter. The PTRAC card of MCNPX code generates an output file which includes all the information needed for tracking the particle. This information includes: (a) the location $(x, y, z)$ of the particle and its related cell and material number, and (b) the ID number, weight, event time, directional cosine, and energy of the particle. The PTRAC output files by positive and negative muons were equal.

\subsection{Analysis of the output file (PTRAC)}

The simulation of penetrating cosmic ray muons through the mountain by $12 \times 10^{9}$ muons was performed. The PTRAC output file is simply an ASCII file including the $x y z$ (Cartesian coordinates), the $u v w$ (directional cosine), the energy, and the event time of the particle (muon) that is being simulated (Zareie 2010 and Tajik et al. 2013). Some information that has been extracted from output file (PTRAC) is presented in Table 1. Data in Table 1 have been extracted from Fig. 1A in the Appendix.

Table 1

A portion of typical data which have been extracted from PTRAC output file

\begin{tabular}{|l|c|c|}
\hline \multicolumn{3}{|c|}{ Extracted information from PTRAC output file } \\
\hline Particle number: 374512 & Entrance to geometry & Exit of geometry \\
\hline Location of particle $(x, y, z)$ & $(-7207.9,-19849,23378)$ & $(45.868,-0.225,-95.656)$ \\
{$[\mathrm{cm}]$} & & \\
Directional cosine & $(0.22962,0.62851,-0.74314)$ & $(0.2323,0.6253,-0.7449)$ \\
Energy of particle $[\mathrm{MeV}]$ & 23000 & 20372 \\
Time of particle $\left[10^{-8} \mathrm{~s}\right]$ & 0.20534 & 105.56 \\
Cell number & 20 & 5 \\
Surface number & 50 & 5 \\
Material number & 1 & 2 \\
\hline
\end{tabular}

Using the output file (PTRAC), the distance that muon travels through matter can be calculated. Practically, this distance is calculated by determination of the muon path through matter using the topographic data. Since the PTRAC file gives the directional cosines, the direction of the entrance of muon to detector and the number of muons as a function of $(\theta, \varphi), N_{\mu}(\theta, \varphi)$, 
in every interval of zenith and azimuthal angles with reference to a line perpendicular to the detector plane can be obtained.

\section{CALCULATION OF THE DENSITY OF MATTER INSIDE THE MOUNTAIN}

There are two possibilities to obtain a two dimensional profile of the density of matter inside the mountain that are mentioned as follows.

\subsection{Using the energy loss of muon inside matter}

A unique relationship exists between the range $\left(\mathrm{in} \mathrm{gr} / \mathrm{cm}^{3}\right)$ and energy of the penetrating cosmic ray muons (Eq. 3). Thus, for both the input and the output muon energy, the range can be calculated, and from the difference between these calculated ranges, the density length is determined (Darijani et al. 2014).

\subsection{The use of relative flux of muons}

The relative intensity of cosmic ray muons transmitted through matter with reference to those directly transmitted through the air is called the relative flux of muons that is introduced as Eq. 7.

$$
n(\theta, \varphi)=\frac{N_{\mu}(\theta, \varphi)}{N_{0}(\theta, \varphi)},
$$

Equation 7 is a unique relation between density length $(X)$ and the intensity of penetrating cosmic ray muons through matter. Figure 5 indicates

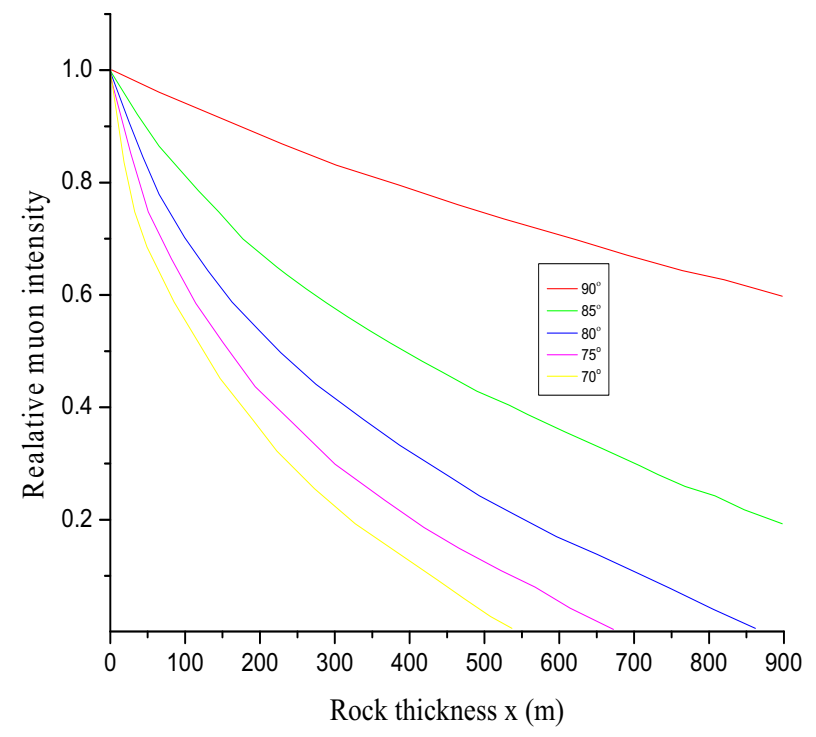

Fig. 5. Relative muon intensity versus rock thickness (Nagamine 2003). 
relative intensity of muons as a function of standard rock thickness. So, for a substance with an unknown density length, the measurement of the muon flux penetrating through the substance with zenith and azimuthal angels uniquely determines its density length (in $\mathrm{gr} / \mathrm{cm}^{3}$ ) (Nagamine 2003). Using the density length, the substance density is determined.

\section{RESULTS}

The simulation was performed with $12 \times 10^{9}$ particles. Because of the symmetry, only $6 \times 10^{9}$ of particles are toward the geometry (Figs. 4 and 6), and the number of muons that were detected after passing through the geometry are about 45720 . Figure 6 shows a two-dimensional picture of production and tracking of muons in the simulation code.

Since cosmic ray muons are constantly irradiating every substance on the Earth at rate of about 70 muon per $\mathrm{m}^{2}$ per min, the performed simulation is equal naturally to the detection of muons for about 35 hours. The limitation of time is due to the limitation of the number of input particles $\left(2 \times 10^{9}\right)$ in MCNPX code.

Because the size of the detector plane $(100 \times 100 \mathrm{~cm})$ is much smaller than the object of interest, the path of each muon can be determined in polar system, as shown in Fig. 4. In this figure the detector plane ( $x z$ plane) is

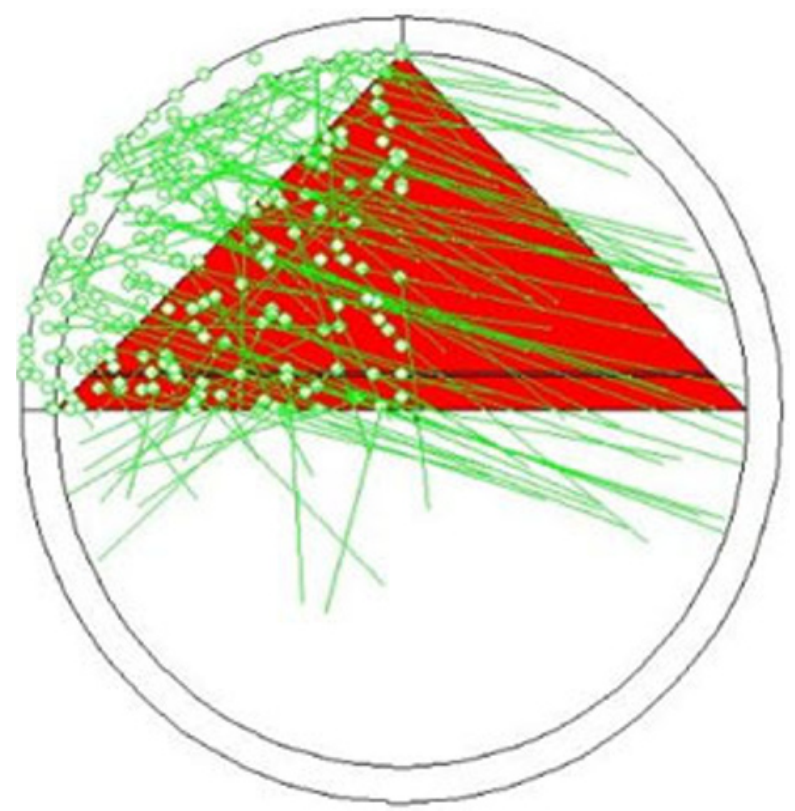

Fig. 6. Two dimensional $y z$ profile of production and tracking of muons. The green dots show muon source points and green lines show muon tracks. 
perpendicular to the $y$ axes, so the zenith and the azimuthal angles are obtained with reference to the $y$ axes. The angle between muon path and $z$ axis is $\theta$, and $\theta_{z}$ (zenith angle) is $90-\theta$ that changes from 0 to 90 degrees (for horizontal and vertical entrance, respectively). The angle between muon path in $x y$ plan and $y$ axis is $\varphi_{x}$ (azimuthal angle) that changes from -90 to 90 degrees. These angles were divided into steps, $\Delta \theta$ and $\Delta \varphi$, and in every interval the number of muons was recorded. A portion of the typical file in which the number of muons has been recorded in every interval has been shown in Table 2.

Table 2

A portion of the counted muons in angle intervals

\begin{tabular}{|c|c|c|c|c|c|c|c|c|c|c|}
\hline \multicolumn{10}{|c|}{ The counted muons in angle intervals $\Delta \theta^{\circ}$ and $\Delta \Phi^{\circ}$} \\
\hline$\Delta \theta^{\circ}$ & $\cdots$ & {$[0,3)$} & {$[3,6)$} & {$[6,9)$} & {$[9,12)$} & {$[12,15)$} & {$[15,18)$} & {$[18,21)$} & {$[21,24)$} & $\cdots$ \\
\hline$\Delta \Phi^{\circ}$ & & & & & & & & & & \\
\hline$\cdots$ & $\cdots$ & $\cdots$ & $\cdots$ & $\cdots$ & $\cdots$ & $\cdots$ & $\cdots$ & $\cdots$ & $\cdots$ & $\cdots$ \\
{$[-69,-66)$} & $\cdots$ & 2 & 14 & 7 & 12 & 11 & 7 & 8 & 0 & $\cdots$ \\
{$[-66,-63)$} & $\cdots$ & 0 & 7 & 4 & 7 & 21 & 18 & 5 & 7 & $\cdots$ \\
{$[-63,-60)$} & $\cdots$ & 3 & 12 & 2 & 14 & 16 & 13 & 1 & 9 & $\cdots$ \\
{$[-60,-57)$} & $\cdots$ & 7 & 4 & 13 & 12 & 29 & 19 & 2 & 13 & $\cdots$ \\
{$[-57,-54)$} & $\cdots$ & 9 & 3 & 15 & 13 & 18 & 31 & 0 & 15 & $\cdots$ \\
{$[-54,-51)$} & $\cdots$ & 12 & 1 & 2 & 10 & 15 & 49 & 13 & 2 & $\cdots$ \\
{$[-51,-48)$} & $\cdots$ & 14 & 4 & 9 & 6 & 22 & 18 & 8 & 2 & $\cdots$ \\
{$[-48,-45)$} & $\cdots$ & 6 & 2 & 3 & 10 & 15 & 18 & 16 & 9 & $\cdots$ \\
{$[-45,-42)$} & $\cdots$ & 11 & 6 & 5 & 9 & 6 & 23 & 25 & 8 & $\cdots$ \\
$\cdots$ & $\cdots$ & $\cdots$ & $\cdots$ & $\cdots$ & $\cdots$ & $\cdots$ & $\cdots$ & $\cdots$ & $\cdots$ & $\cdots$ \\
\hline
\end{tabular}

Using the output file (PTRAC), two dimensional profiles of the number of muons and density of matter that muons penetrate through it were plotted and have been shown in Figs. 7-10. Figures 7 and 8 indicate the two dimensional profiles of the number of penetrating particles through the mountain without and with a layer of coal, respectively. The two dimensional profiles of the density of the mountain without and with a layer of coal, that muons penetrate through it, have been shown in Figs. 9 and 10. A comparison of Figs. 9, 10a, and 10b shows the existence of the region with lower density (the layer of coal) in the mountain. Figure 10 shows that around $\varphi_{x}=0$ (parallel to the $y$ axis) and for $\theta_{z}$ between 0 and 5 degrees, there is a matter that its density is less than the density of the standard rock. By this approach, the layers with minimum thickness of about $2.5 \mathrm{~m}$ are identified. 


\section{xz-number}

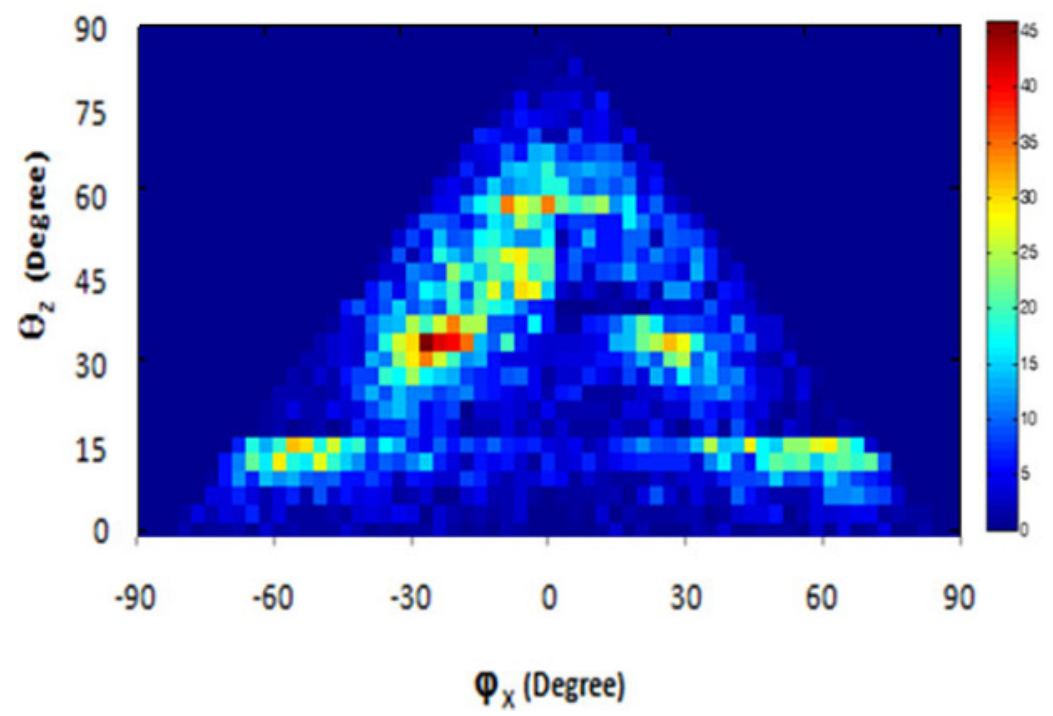

Fig. 7. Two dimensional profile from number of particles in the $x z$ plan without any layer of coal.

\section{xz-number}

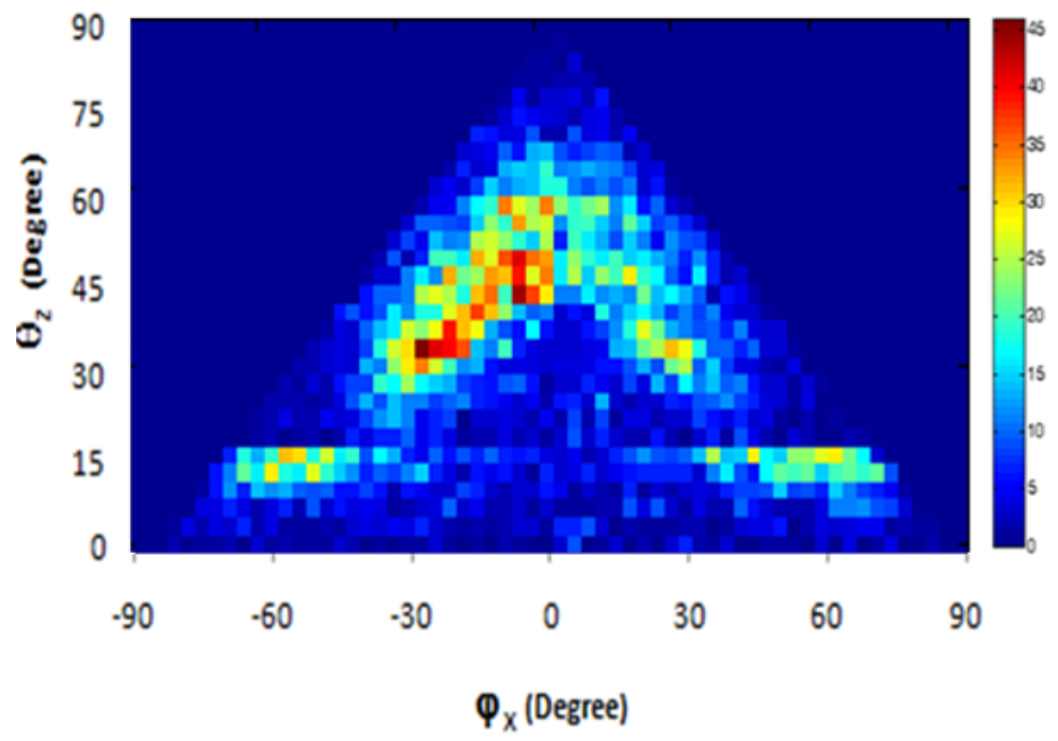

Fig. 8. Two dimensional profile from number of particles in the $x z$ plan with a layer of coal. 


\section{xz-Profile}

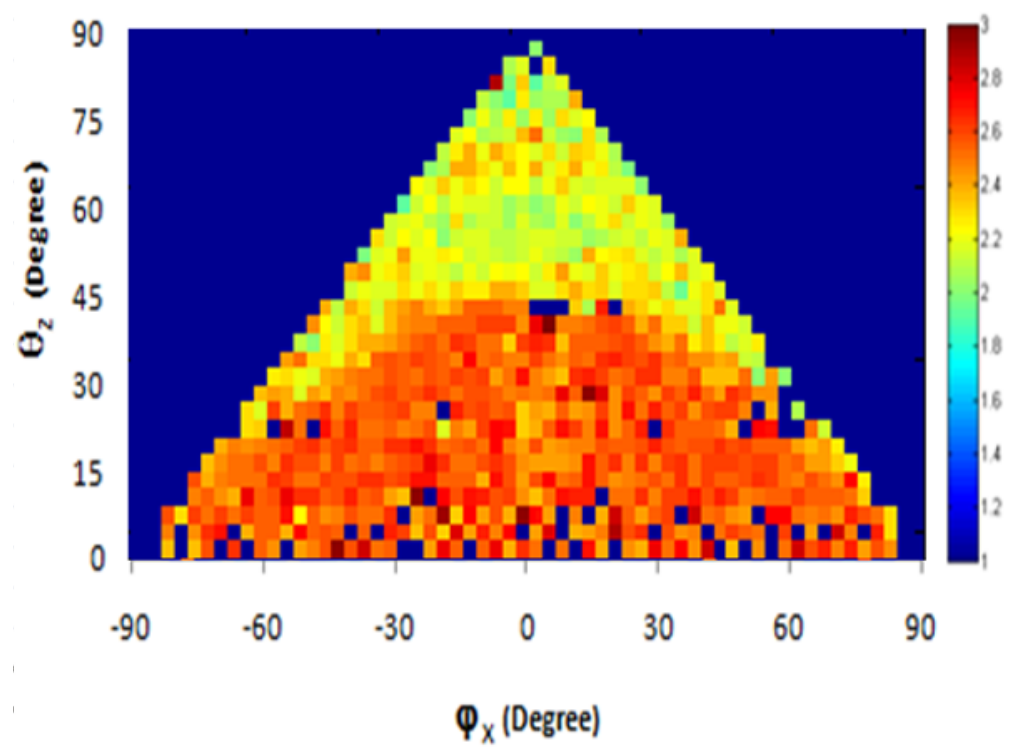

Fig. 9. Two dimensional profile from density of mountain in the $x z$ plan without any layer of coal.

\section{xz-Profile}
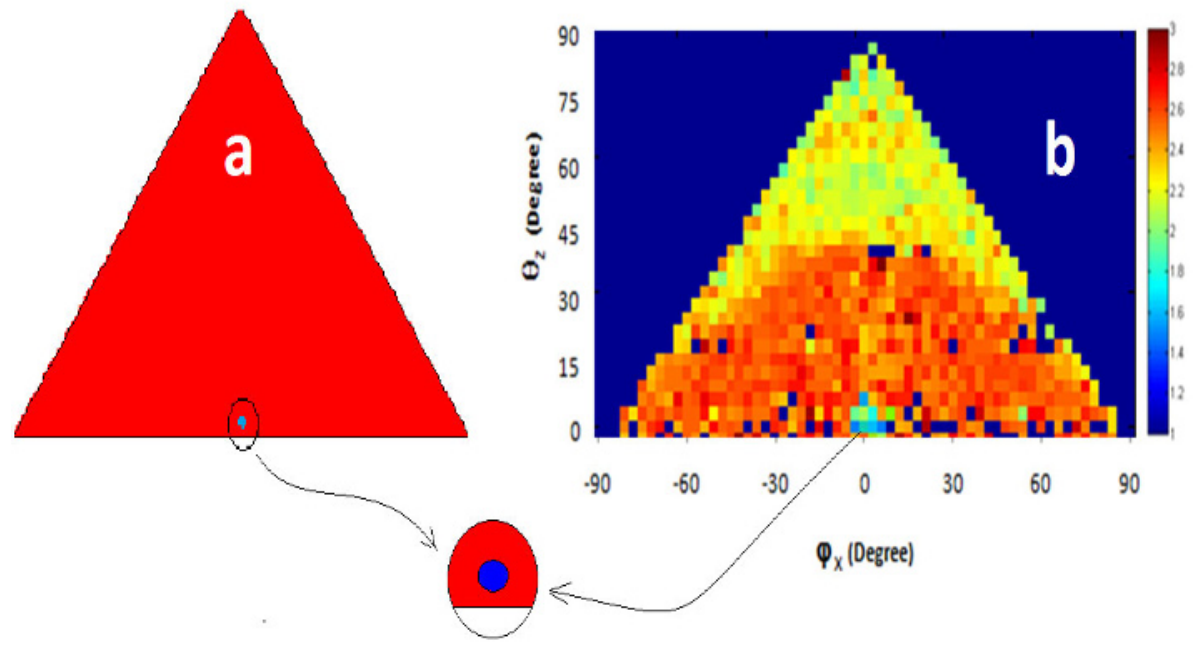

Fig. 10: (a) Two dimensional $x z$ profile of geometry that the code provides (the blue circle shows the $x z$ profile of cylindrical coal layer), and (b) two dimensional profile from density of mountain in $x z$ plan with a layer of coal due to MCNPX simulation results. 


\section{CONCLUSION}

Very high energy muons are most suitable for measuring the density profile of a large scale substance like a mountain. These muons also are used for exploration of mines. The spatial resolution to determine the internal intensity of a mountain depends on not only the spatial resolution of detector but also the distance of the detector from the center of mountain. In this study, the detector was placed inside the mountain (Fig. 4) that increased the spatial resolution and decreased the interest area for imaging. Using many detectors at different altitudes of a mountain, radiography can be done from more parts of mountain, the spatial resolution can be increased, and the exact location of coal layers in a mountain can be determined. Figures 7 to 10 are the results of using one detector.

\section{Appendix}

\section{Interpretation of data in PTRAC output file}

PTRAC output file is an ASCII file which includes all information required for tracking a particle. This information includes: (a) the location $(x, y, z)$ of the particle and its related cell, and material number (b) the ID number, weight, event time, directional cosine, and energy of the particle. A portion of typical PTRAC output has been shown in Fig. 1A.

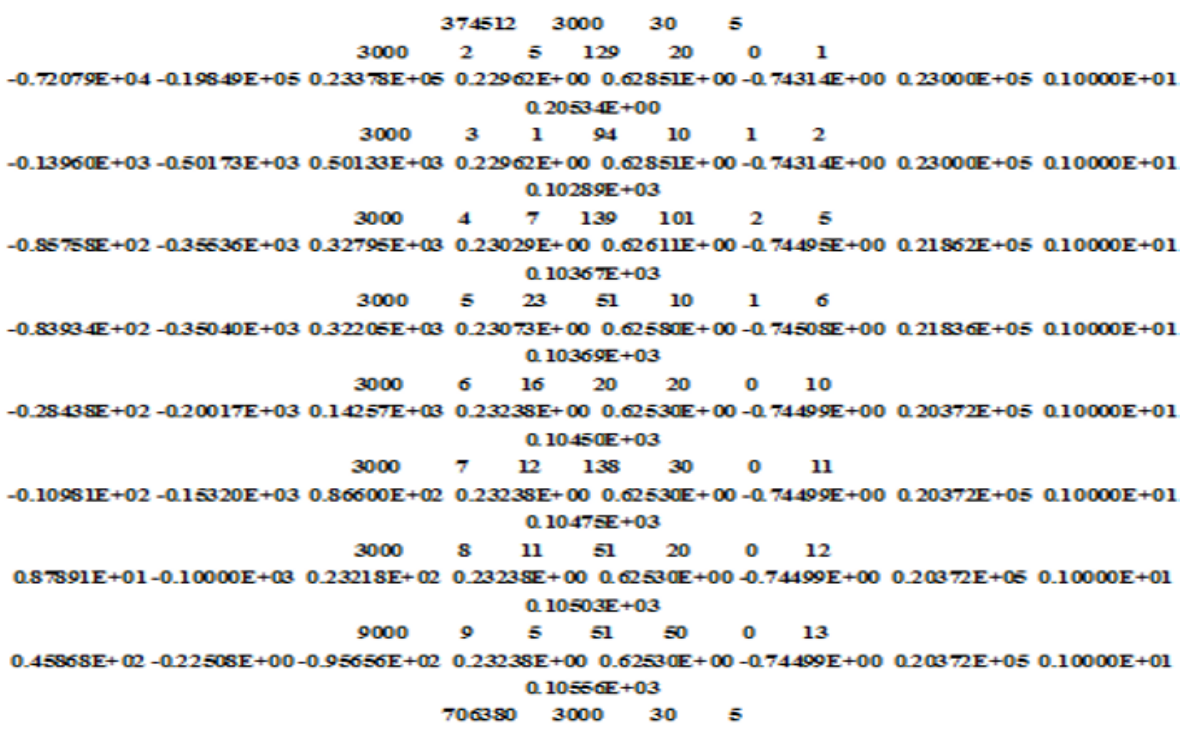

Fig. 1A. A portion of typical PTRAC output. 
The first number of the first line (i.e., 374512) is an ID number for the particle, number 3000 indicates the entrance of the particle to the geometry, number 30 is the number of the cell that has been filtered, and number 5 determines the entrance surface of the source particles in the geometry. In the second line, numbers 5 and 20 show that the particle has been entered from surface 5 into cell 20 , number 0 is the material number, number 2 is the line number, and number 129 is the angle between the particle path and the line perpendicular to the surface 5. In the third line, the first six numbers are Cartesian coordinates $(x, y, z)$ and direction cosines $(u, v, w)$ of the entered particle, respectively. Numbers $0.23000 \mathrm{E}+05,0.10000 \mathrm{E}+01$, and $0.20534 \mathrm{E}+00$ in the third line are the energy, weight, and event time of the particle, respectively. In line 16, number 9000 characterizes the exit of the particle from the geometry.

\section{References}

Allkofer, O.C., K. Carstensen, G. Bella, W.D. Dau, H. Jokisch, G. Klemke, Y. Oren, and R.C. Uhr (1981), Muon spectra from DEIS up to $7 \mathrm{TeV}$. In: Proc. 17th Int. Cosmic Ray Conference, 13-25 July 1981, Paris, France, Vol. 10, 321324.

Alvarez, L.W., J.A. Anderson, F. El Bedwei, J. Burkhard, A. Fakhry, A. Girgis, A. Goneid, F. Hassan, D. Iverson, G. Lynch, Z. Miligy, A.H. Moussa, M. Sharkawi, and L. Yazolino (1970), Search for hidden chambers in the pyramids, Science 167, 3919, 832-839, DOI: 10.1126/science.167.3919. 832.

Bektasoglu, M., H. Arslan, and D. Stanca (2012), Simulations of muon flux in slanic salt mine, Adv. High Energy Phys. 2012, 751762, DOI: 10.1155/2012/ 751762.

Bugaev, E.V., Y.D. Kotov, and I.L. Rosental (1970), Cosmic Muons and Neutrinos, Atomizdat, Moscow, $320 \mathrm{pp}$.

Darijani, R., A. Negarestani, M.R. Rezaie, J. Fatami, and A. Akhond (2014), Formulation of muon range $0-100 \mathrm{TeV}$ and transmission through lead, Indian $J$. Pure Appl. Phys. 52, 1, 7-12.

Gaisser, T.K. (1990), Cosmic Rays and Particle Physics, Cambridge University Press, Cambridge.

Lesparre, N., D. Gibert, J. Marteau, Y. Déclais, D. Carbone, and E. Galichet (2010), Geophysical muon imaging: feasibility and limits, Geophys. J. Int. 183, 3, 1348-1361, DOI: 10.1111/j.1365-246X.2010.04790.x.

Malmqvist, L., G. Jönsson, K. Kristiansson, and L. Jacobsson (1979), Theoretical studies of in-situ rock density determinations using underground cosmic- 
ray muon intensity measurements with application in mining geophysics, Geophysics 44, 9, 1549-1569, DOI: 10.1190/1.1441026.

Nagamine, K. (2003), Introductory Muon Science, Cambridge University Press, Cambridge.

Nagamine, K., M. Iwasaki, K. Shimomura, and K. Ishida (1995), Method of probing inner-structure of geophysical substance with the horizontal cosmic-ray muons and possible application to volcanic eruption prediction, Nucl. Instrum. Meth. Phys. Res. A 356, 2-3, 585-595, DOI: 10.1016/01689002(94) 01169-9.

Pelowitz, D.B. (ed.) (2008), MCNPX user's manual, version 2.6.0, LA-CP-07-1743, Los Alamos National Laboratory, Los Alamos, USA.

Tajik, M., N. Ghal-Eh, G.R. Etaati, and H. Afarideh (2013), Modeling NE213 scintillator response to neutrons using an MCNPX-PHOTRACK hybrid code, Nucl. Instrum. Meth. Phys. Res. A 704, 104-110, DOI: 10.1016/j.nima. 2012.12.001.

Tanaka, H., K. Nagamine, N. Kawamura, S.N. Nakamura, K. Ishida, and K. Shimomura (2003), Development of a two-fold segmented detection system for near horizontally cosmic-ray muons to probe the internal structure of a volcano, Nucl. Instrum. Meth. Phys. Res. A 507, 3, 657-669, DOI: 10.1016/ S0168-9002(03)01372-X.

Tanaka, H.K.M., T. Nakano, S. Takahashi, J. Yoshida, and K. Niwa (2007), Development of an emulsion imaging system for cosmic-ray muon radiography to explore the internal structure of volcano, Mt. Asama, Nucl. Instrum. Meth. Phys. Res. A 575, 3, 489-497, DOI: 10.1016/j.nima.2007.02.104.

Thompson, M.G., and M.R. Whalley (1975), The production spectra of the parents of vertical cosmic ray muons, J. Phys. G 1, 48-50, DOI: 10.1088/0305$4616 / 1 / 6 / 004$

Zareie, S.H. (2010), A Monte Carlo simulation of the Compton camera, M.Sc. Thesis, San Diego State University, San Diego, USA.

Received 19 August 2014

Received in revised form 25 April 2015

Accepted 2 June 2015 